\title{
Nausea and vomiting in pregnancy - not always what it seems
}

\author{
Edral A., Martins R., Oliveira C., Ferreira A. \\ Centro Hospitalar Universitário do Algarve (CHUA) - Faro
}

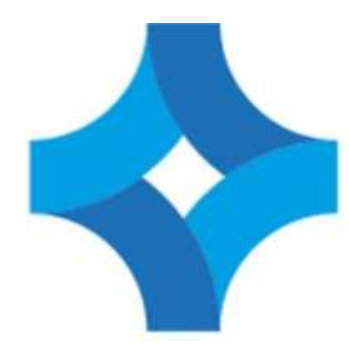

Centro

Hospitalar

Universitário

do Algarve

\section{Problem Statement}

Nausea and vomiting are common symptoms in pregnancy, affecting 50-80\% of pregnant women. However, when there are atypical signs and/or symptoms, there should be a more in-depth examination, in order to exclude other causes and determine if they are associated with pregnancy or not.

\section{Case Report}

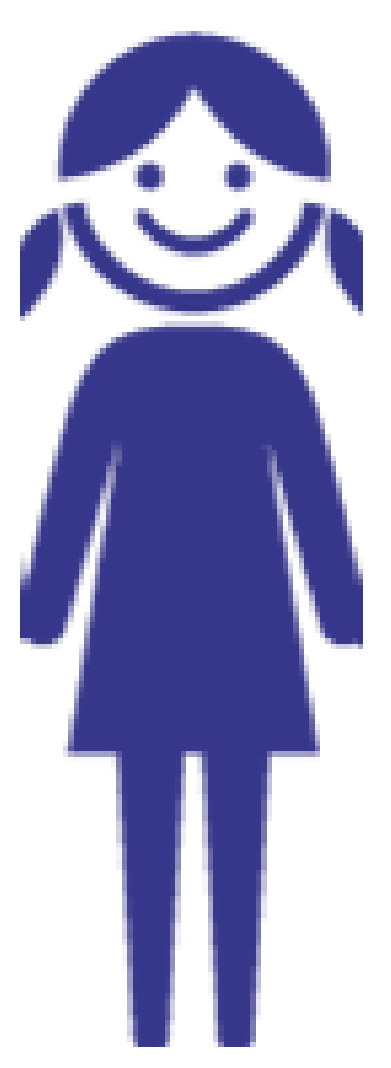

28 years old

Raynaud Syndrome Unremarkable familial history

Last Menstrual Period? 2G2P uneventful pregnancies
- Nausea and vomiting 3 days ago

- Normal physical examination, including neurological examination

- Blood analysis: $\beta-\mathrm{hCG}=15000$ (non-pregnant $<5 \mathrm{mU} / \mathrm{mL}$ ), remaining values were normal

- Obstetric ultrasound: single intrauterine pregnancy 6 weeks

- Medication: Doxylamine + Dicloverine + Pyridoxine

- Persistent frontal headache, vomiting, anxiety, marital problems, lost of will to perform everyday tasks

- Signs of widespread malaise, excessive sweating, pallor but conscious and cooperative, oriented

- Normal blood analysis

- Decision: hospitalization for surveilance and symtomatic therapy

- At the first day: generalized tonic-clonic seizure (lasting 2 minutes)

- Glasgow Coma Scale Score $=8$

- Blood analysis: metabolic acidosis with hyperlactacidemia and hypokalemia

- Decision (multidiscilpinar): replacement therapy + phenytoin + emergent brain CT scan

- During the CT-scan: cardio-respiratory arrest $\rightarrow$ Advanced life support $\rightarrow$ heart rate was recovered

- Brain tumor (figure 1)

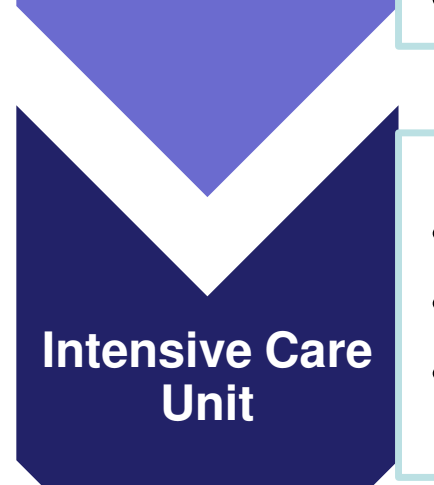

- Pharmacological measures to reduce brain swelling and analgesia

- 10h later she had a second cardio-respiratory arrest episode which was not reverted

- Family refused a body autopsy

The most common neurologic complications in pregnancy are those secondary to arterial-venous malformations, aneurysms and preeclampsia. Brain tumors are rare and may present with symptoms similar to those of normal pregnancy or its complications.

Nausea and vomiting associated with normal pregnancy tend to improve later in pregnancy, on the opposite those associated with brain tumors, may arise later, tend to worsen gradually, and may coexist with symptoms such as headache, visual disturbances or focal neurological deficits.

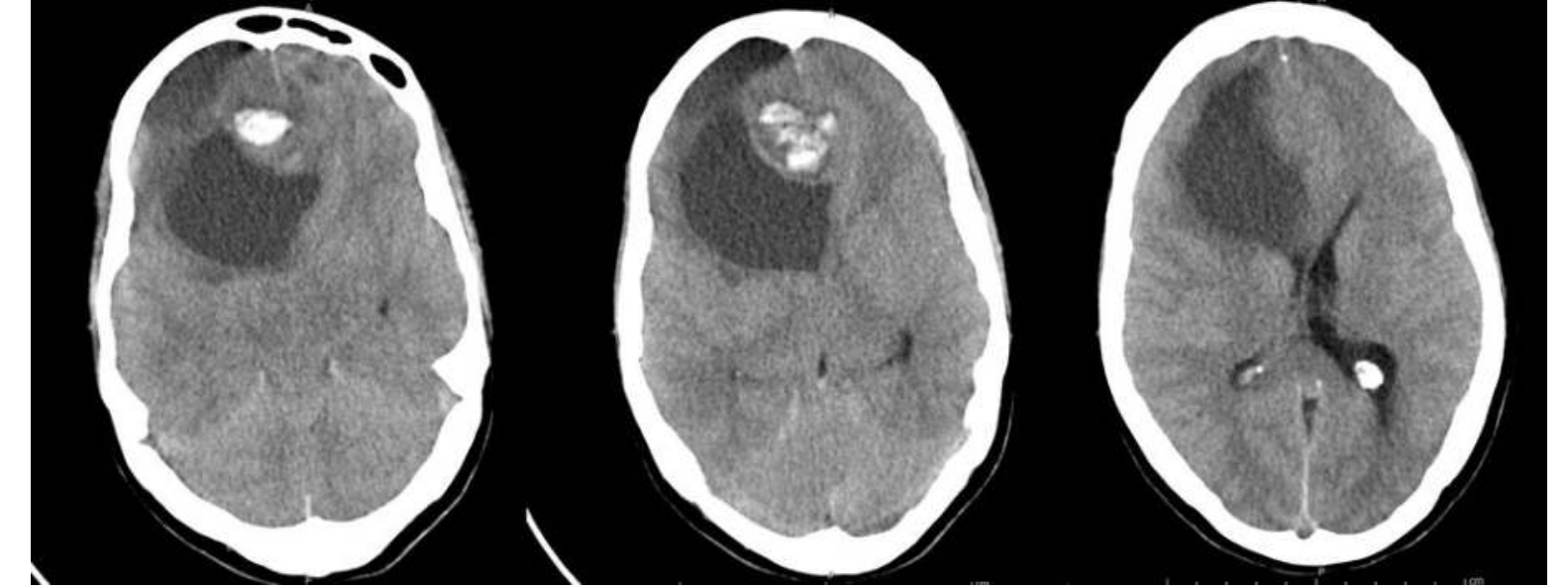

Figure 1: expansive right frontal-basal lesion with hemorrhagic areas and an extensive cystic area, causing a mass effect with compression of the right frontal lateral ventricle horn, slight midline shift and cerebral cistern deletion

It is known that pregnancy does not modify the incidence of brain tumors and their prevalence reflects the same age group in the general population. However, hemodynamic and hormonal changes during the pregnancy may accelerate tumor growth at this stage, with the possible worsening of the signs and symptoms.

\section{Conclusion}

This case displays the importance of a careful and critical approach to every pregnant woman. One should consider several differential diagnoses and also to pay attention to the atypical signs and symptoms. The multidisciplinary discussion is also of crucial importance. In the case of brain tumors, early diagnosis can make a difference in the prognosis. 